\title{
Autophagy Exacerbates the Lysosomal Dysfunction of Late-Stage Atherosclerosis.
}

\author{
Jerome $\mathrm{WG}^{1,2}$, Netherland-Van Dyke $\mathrm{CD}^{1}$, Romer $\mathrm{CE}^{1}$ \\ 1. Pathology, Microbiology and Immunology, Vanderbilt University, Nashville, USA \\ 2. Cancer Biology, Vanderbilt University, Nashville, USA
}

Cardiovascular disease is the number one killer in the developed world. Atherosclerosis underlies much of the cardiovascular problems. Atherosclerosis is a progressive thickening of the artery wall due to accumulation of excess lipid and cells. Lipid-engorged macrophages, called foam cells, are a key component of atherosclerotic lesions and understanding exactly how lipid is metabolized in these cells is a critical area of atherosclerosis research.

Cholesterol is the primary lipid found in foam cells. The cholesterol is derived from circulating low density lipoproteins (LDL) where it occurs as cholesteryl esters (CE); a cholesterol molecule esterified to a long chain fatty acid (FA). In the artery wall, macrophages endocytose LDL and deliver the CE to lysosomes where it can be hydrolyzed to free cholesterol (FC) and FA. Normally, the FC can be transported out of the lysosome and become part of membranes while excess FC is reesterified to FA in rER to form CE that accumulates in cytoplasmic lipid inclusions. In contrast to this normal situation, in late stages of atherosclerosis, excess FC is not cleared and so the sterol concentration in lysosomes becomes so excessive that lysosomal function is inhibited. This lysosomal dysfunction disrupts normal cellular metabolism and can lead to cell death, inflammation, and other pathologies.

Autophagy is a mechanism by which cells target internal components to lysosomes for recycling. Recently, this pathway has been implicated in the metabolism of excess cytoplasmic sterol stores in foam cells in early lesions. However, we reasoned that if this pathway remained active in late stage lesions, it would target sterol to inactive lysosomes and thus exacerbate the lysosomal sterol accumulation. To test this, we incubated THP-1 macrophages with 35 ug protein/ml aggregated LDL (aggLDL) for 6 days (+3 days in media alone) to produce sterol-mediated lysosomal dysfunction. Control cells were incubated in media alone for 9 days.

To investigate the effect of this treatment on autophagy, we analyzed cellular LC3, an autophagic marker. When cytoplasmic LC3-I is activated by association with phosphatidylethanolamine to form LC3-II, it binds autophagosome membranes and targets cargo for lysosome degradation. Thus, changes in the total amount of LC3 and the ratio of LC3-I to LC3-II are sensitive markers for autophagic flux. By western blot we found a significant increase in both LC3-I and LC3-II levels with sterol accumulation indicative of increases in autophagy. Fluorescence immunomicroscopy (Figure 1) indicated a shift in LC3 location from cytoplasmic to lysosomal after 6 days of treatment with aggLDL. This was confirmed by colocalization analysis (Figure 2A) showing that aggLDL loading produced a significant increase in the association of LC3 with the lysosome marker, LAMP-1. This suggests an increased targeting of autophagic vesicles to lysosomes and/or an inhibition of degradation of autophagic material. We also found an increased association of LC3 with the lipid droplet marker, adipophilin (Figure 2B) indicating specific targeting of cytoplasmic lipid, including sterol, to the autophagic pathway. Finally, fluorescence colocalization of LAMP-1 with adipophilin (Figure 2C) showed a dramatic increase (>3 fold) in adipophilin within lysosomes; suggesting that autophagy was mobilizing lipid droplet contents to lysosomes. 
Measuring cellular and lysosomal sterol levels, we found that despite the increase in autophagy, the cells continued to accumulate rather than clear cholesterol. Thus our data are consistent with the hypotheses 1) that cellular sterol accumulation activates lipid-specific autophagy and 2) that this targeting does not result in sterol clearance, rather the increased delivery of sterol to dysfunctional lysosomes merely exacerbated the lysosomal dysfunction [1].

[1] The authors acknowledge funding from NIH, NHLBI RO1 HL086746 (WGJ); AHA 12GRNT12070011 (WGJ); AHA Postdoctoral Fellowship 13POST14240000 (CDN).

Figure 1

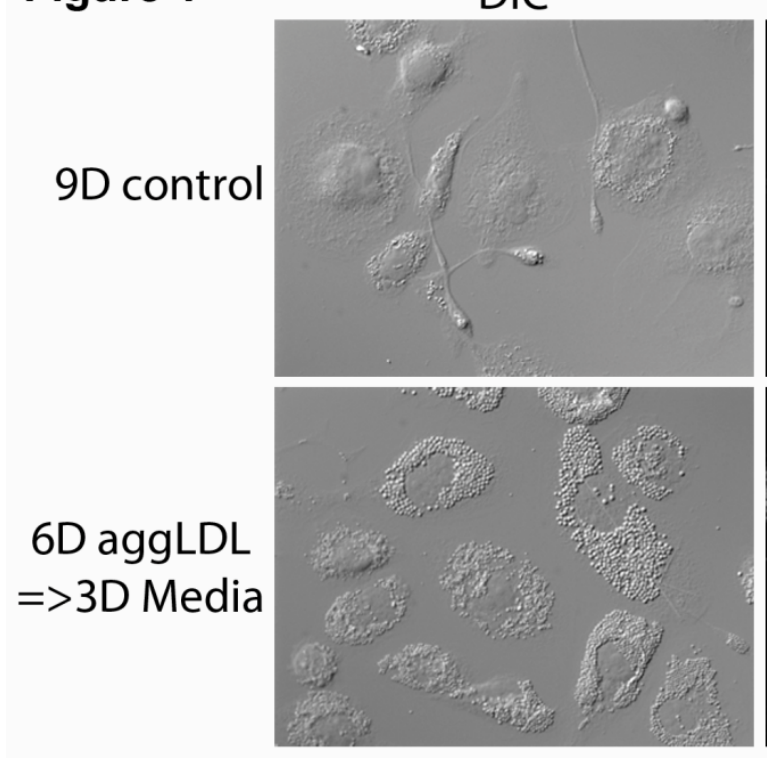

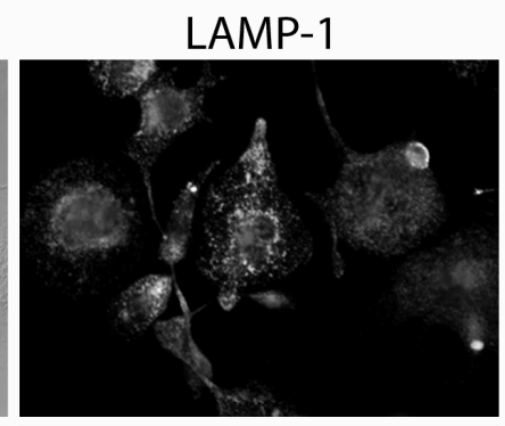
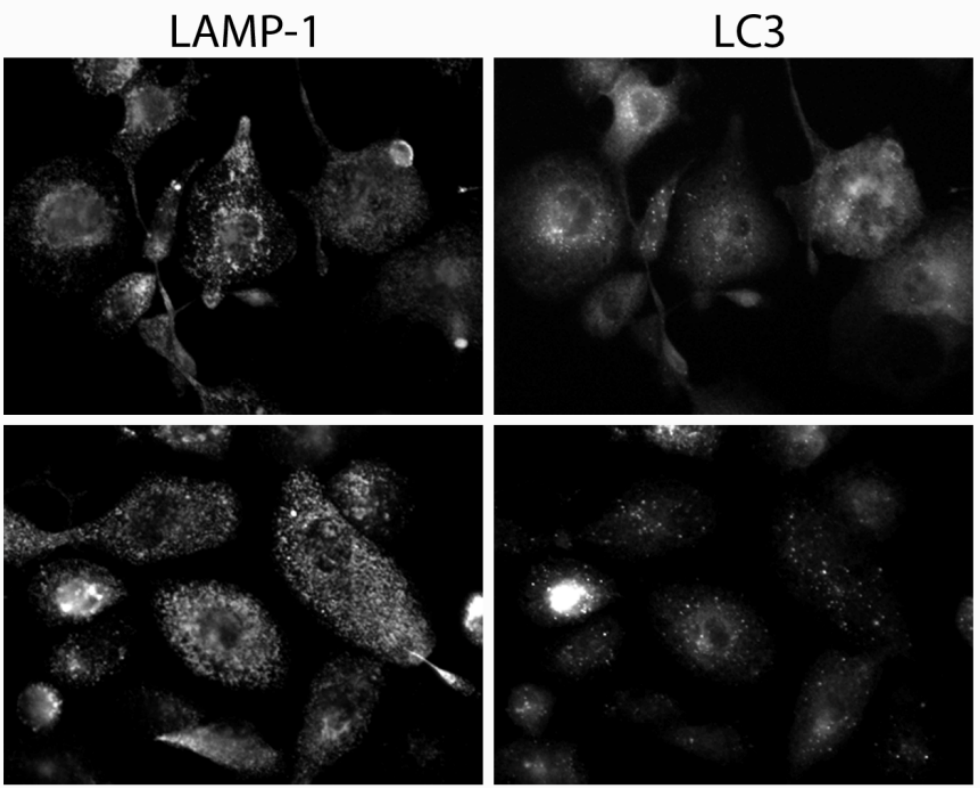

Figure 1. Effect of aggLDL treatment on cellular lipid and LAMP-1 and LC3 location within macrophages.
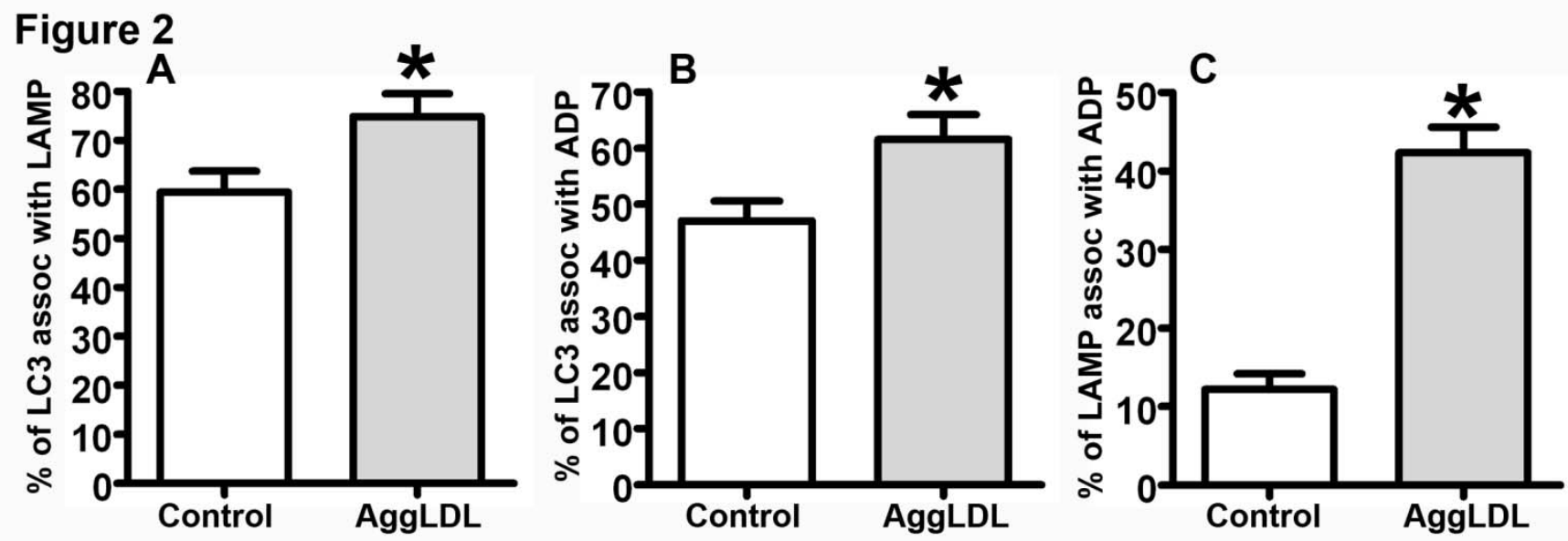

Figure 2. Quantification of changes in colocalization of LAMP-1, LC3 and Adipophilin (ADP) when cells are incubated for 6 days with aggLDL and chased with media alone for 3days. 